\title{
Optimal Control of Pseudoparabolic Variational Inequalities Involving State Constraint
}

\author{
Youjun $\mathrm{Xu}^{1,2}$ and Shu Zhou ${ }^{1}$ \\ ${ }^{1}$ School of Mathematics and Physics, University of South China, Hunan 421001, China \\ ${ }^{2}$ School of Mathematical Sciences, Fudan University, Shanghai 200433, China \\ Correspondence should be addressed to Youjun Xu; youjunxu@163.com
}

Received 5 March 2013; Accepted 27 May 2013; Published 20 January 2014

Academic Editor: Victor Kovtunenko

Copyright (c) $2014 \mathrm{Y}$. Xu and S. Zhou. This is an open access article distributed under the Creative Commons Attribution License, which permits unrestricted use, distribution, and reproduction in any medium, provided the original work is properly cited.

We establish the necessary condition of optimality for optimal control problem governed by some pseudoparabolic differential equations involving monotone graphs. Some approximating control process and examples are given.

\section{Introduction}

We will study the following optimal control problem governed by nonlinear pseudoparabolic variational inequalities of the following form:

$$
\begin{gathered}
\frac{d M y}{d t}+A y+\beta(y) \ni B u \text { a.e in }(0, T), \\
y(0)=y_{0},
\end{gathered}
$$

with the state constraint

$$
F(y(\cdot)) \subset S
$$

The pay-off function is given by

$$
L(y(\cdot), u(\cdot))=\int_{0}^{T}[g(t, y(\cdot))+h(u(\cdot))] d t,
$$

where $Q=\Omega \times(0, T), \Omega \subset \mathbb{R}^{N}$ is a bounded domain with smooth boundary. tions.

For the problem (1)-(3), we have the following assump-

(H1) $M$ is a selfadjoint operator in $H=€^{2}(\Omega)$ with $D(M) \subset D(A+\beta)$ such that for every $y \in D(M)$,

$$
(M y, y) \geq a|y|^{2}, \quad a>0 .
$$

Throughout in the sequel, we will denote by $|\cdot|$ and $(\cdot, \cdot)$ the norm and the scalar product of $H$, respectively. The norm of the control set $U$ will be denoted by $|\cdot|_{U}$ and the scalar product $\langle\cdot, \cdot\rangle$, respectively. $D(M), D(A+\beta)$ denote the domain of operator $M, A+\beta$, respectively.

(H2) $V \subset H$ is a real Hilbert space such that $V$ is dense in $H$ and $V \subset H \subset V^{\prime}$ algebraically and topologically, where $V^{\prime}$ is the dual of $V$. Further, the injection of $V$ into $H$ is compact.

$A: V \rightarrow V^{\prime}$ is a linear continuous and symmetric operator from $V$ to $V^{\prime}$ satisfying the coercivity condition

$$
(A y, y) \geq w\|y\|_{V}^{2}+\alpha|y|_{H}^{2} \quad \forall y \in V,
$$

where $w>0$ and $\alpha \geq 0$.

(H3) $\beta$ is a maximal monotone graph in $\mathbb{R} \times \mathbb{R}$ with $0 \in$ $\beta(0)$. Let $\phi(y): H \rightarrow \mathbb{R}=(-\infty,+\infty]$ be the lower semicontinuous convex function defined by $\phi(y)=$ $\int_{\Omega} j(y) d x$, where $j: \mathbb{R} \rightarrow \overline{\mathbb{R}}$ is such that $\partial j=\beta$. Moreover,

$$
\left(A y, \beta_{\epsilon}(y)\right) \geq 0 \quad \forall y \in D(A), \epsilon>0,
$$


where $\beta_{\epsilon}(r)=\epsilon^{-1}\left(r-(1+\epsilon \beta)^{-1} r\right)$ for all $\epsilon>0, r \in \mathbb{R}$. For every $\xi \in \beta$, there exists a constant $c$ such that

$$
|\xi(s)| \leq c\left(1+|s|^{p+1}\right)
$$

where $0 \leq p \leq 2 /(N-2)$ if $N>2$ and $0 \leq p<$ $+\infty$ if $N=1,2 . \partial j$ denotes the generalized Clarke subdifferential of the function $j$.

(H4) $B$ is a linear continuous operator from a real Hilbert space $U$ to $H$.

(H5) Let $\mathbb{Z}$ be a Banach space with the dual $\mathbb{Z}^{*}$ strictly convex. $S \subset \mathbb{Z}$ is a closed convex subset with finite codimensionality [1-3]. $F: L^{2}(0, T ; V) \rightarrow \mathbb{Z}$ is of class $C^{1}$.

(H6) The functional $h: U \rightarrow \overline{\mathbb{R}}$ is convex and lower semicontinuous (l. s. c), such that

$$
h(u) \geq c_{1}|u|_{U}^{2}+c_{2}
$$

where $c_{1}>0, c_{2} \in \mathbb{R}$, for all $u \in U$.

(H7) $g:[0, T] \times H \rightarrow \mathbb{R}^{+}$is measurable in $t$, and for every $\delta>0$, there exists $L_{\delta}>0$ independent of $t$ such that $g(t, 0) \in L^{\infty}(0, T)$ and

$$
\begin{gathered}
\left|g\left(t, y_{1}\right)-g\left(t, y_{2}\right)\right| \leq L_{\delta}\left|y_{1}-y_{2}\right|_{H} \\
\forall t \in[0, T], \\
\left|y_{1}\right|_{H}+\left|y_{2}\right|_{H} \leq \delta .
\end{gathered}
$$

Remark 1. Note that, by (H3), system (1) is equivalent to

$$
\begin{gathered}
\frac{d M y}{d t}+A y+\partial \phi(y(t)) \ni B u \quad \text { a.e } t \in(0, T), \\
y(0)=y_{0} .
\end{gathered}
$$

As we know, by Barbu [4] (see Chapter 4) and Theorem 1.1 of [5], we have the following.

Lemma 2. Let (H1)-(H4) hold. Then, for any $y_{0} \in D(M) \cap V$, $u \in L^{2}(0, T ; U)$, (1) admits a unique solution $y(x, t)$ satisfying

$$
y \in W^{1,2}([0, T] ; H) \cap L^{2}(0, T ; D(M) \cap V) \cap C([0, T] ; H) .
$$

Now we formulate the optimal control problems as follows.

Let $A_{a d}=\left\{(y, u) \in W^{1,2}([0, T] ; H) \cap C([0, T] ; H) \cap\right.$ $L^{2}(0, T ; D(M)) \times L^{2}(0, T ; U) \mid y$ is the solution of (10) with (2)\}.

We will find

$$
\min L(y, u) \text { over }(y, u) \in A_{a d} .
$$

Recently, some optimal control problems governed by pseudoparabolic equations have already been discussed. Linear optimal control problems for pseudoparabolic equations were considered by many authors (cf. [6-12]). However, these problems studied in [7-12] do not involve state constraints and maximal monotone graph. On the other hand, optimal control problems governed by some parabolic variational inequalities (cf. [4, 13-19]) have already been discussed. Li and Yong [1] studied the maximal principle for optimal control governed by some nonlinear parabolic equations with two point boundary (time variable) state constraints. In Cases' work [20], the state constraint was considered, but the state equation did not involve monotone graph. He [21] studied the optimal control problems involving some special maximal monotone graph (Lipschitz continuous) with state constraint. Wang $[2,3]$ also discussed the optimal control problem governed by the state equation involving some maximal monotone graph.

The present work in this paper considers the optimal control problem governed by the pseudoparabolic equations which is different from what they discussed in $[7-9,12]$, with the state constraints which is similar to those in $[3,4,21]$.

The plan of this paper is as follows. Section 2 gives an approximating control process. In Section 3, we state and prove the necessary conditions on optimality for the problem (P). In Section 4, some examples are given.

\section{The Approximating Control Process}

Let $\left(y^{*}, u^{*}\right)$ be optimal for the problem $(\mathrm{P})$. Then

$$
\begin{gathered}
\frac{d M y^{*}}{d t}+A y^{*}+\partial \phi\left(y^{*}(t)\right) \ni B u^{*} \text { a.e } t \in(0, T), \\
y^{*}(0)=y_{0}
\end{gathered}
$$

with

$$
\begin{gathered}
F\left(y^{*}\right) \in S, \\
L\left(y^{*}, u^{*}\right)=\inf L(y, u) \quad \text { over }(y, u) \in A_{a d} .
\end{gathered}
$$

From a perturbation theorem for $\mathrm{m}$-accretive operators ([22], Lemma 5) and (H2), (H3), we easily know that $C(=A+$ $\beta)$ is $\mathrm{m}$-accretive in $H$.

Now consider the following approximating equation:

$$
\begin{gathered}
\frac{d M y}{d t}+C_{\epsilon} J_{\epsilon}^{M} y=B u \quad \text { a.e in }(0, T), \\
y(0)=y_{0},
\end{gathered}
$$

where $C_{\epsilon}=\epsilon^{-1}\left(I-J_{\epsilon}^{C}\right)$ and $J_{\epsilon}^{C}=(I+\epsilon C)^{-1}$. By Lemma 2, for any $y_{0} \in D(M) \cap V, u \in L^{2}(0, T ; U)$, (14) has a unique solution in $W^{1,2}((0, T] ; H) \cap C([0, T] ; H) \cap L^{2}(0, T ; V)$.

Besides, we have the following result on (14).

Lemma 3. For $\epsilon>0$ given, let $u_{n} \in L^{2}(0, T ; U), u_{n} \rightarrow$ $\tilde{u}$ weakly in $L^{2}(0, T ; U)$, and $\tilde{y}, y_{n}$ the solutions of (14) corresponding to $\tilde{u}$ and $u_{n}$, respectively. Then, there exists some subsequence of $\left\{y_{n}\right\}$, still denoted by itself, such that $y_{n} \rightarrow \tilde{y}$ strongly in $C([0, T] ; H) \cap L^{2}(0, T ; V)$. 
Proof. Multiplying (14) by $J_{\epsilon}^{M} y_{n}(t)$ and using the selfadjointness of $M$, we see the following:

$$
\begin{gathered}
\frac{d}{d t}\left|M_{\epsilon}^{1 / 2} y_{n}(t)\right|^{2}+2\left(C_{\epsilon} J_{\epsilon}^{M} y_{n}(t), J_{\epsilon}^{M} y_{n}(t)\right) \\
=2\left(B u_{n}, J_{\epsilon}^{M} y_{n}(t)\right) .
\end{gathered}
$$

Then (H1)-(H3) yield

$$
\frac{d}{d t}\left|M_{\epsilon}^{1 / 2} y_{n}(t)\right|^{2} \leq c\left|M_{\epsilon}^{1 / 2} y_{n}(t)\right|^{2}+c\left|B u_{n}\right|^{2},
$$

where $M_{\epsilon}=\epsilon^{-1}\left(I-J_{\epsilon}^{M}\right)$. Integrating the above inequality from 0 to $t(t \in(0, T])$ and using Gronwall's inequality, we see the following:

$$
\left|M_{\epsilon}^{1 / 2} y_{n}(t)\right|^{2} \leq c \quad \forall t \in[0, T] .
$$

Note that from (H1), $M$ has a bounded inverse operator on $H$ and

$$
a\left|J_{\epsilon}^{M} y_{n}(t)\right|^{2} \leq\left|M^{1 / 2} J_{\epsilon}^{M} y_{n}(t)\right|^{2} \leq\left|M_{\epsilon}^{1 / 2} y_{n}(t)\right|^{2} .
$$

Together (17) and (18), we have the following:

$$
\left|J_{\epsilon}^{M} y_{n}(t)\right|^{2} \leq c \quad \forall t \in[0, T] .
$$

Since $|v|^{2}=\epsilon\left(M_{\epsilon} v, v\right)+\left(J_{\epsilon}^{M} v, v\right)$ for every $v \in H$, taking into account (17) and (19), we have the following:

$$
\left|y_{n}(t)\right|^{2} \leq c \quad \forall t \in[0, T] .
$$

Multiplying (14) by $M_{\epsilon} J_{\epsilon}^{M} y_{n}(t)$, we see

$$
\begin{gathered}
\frac{d}{d t}\left|M_{\epsilon} y_{n}(t)\right|^{2}+2\left(C_{\epsilon} J_{\epsilon}^{M} y_{n}(t), M_{\epsilon} J_{\epsilon}^{M} y_{n}(t)\right) \\
=2\left(B u_{n}, M_{\epsilon} J_{\epsilon}^{M} y_{n}(t)\right) .
\end{gathered}
$$

Then we get the following:

$$
\frac{d}{d t}\left|M_{\epsilon} y_{n}(t)\right|^{2} \leq c\left|M_{\epsilon} y_{n}(t)\right|^{2}+c\left|u_{n}\right|_{U}^{2} .
$$

Applying Gronwall's inequality to the above inequality and noting that $\left\{u_{n}\right\}$ is bounded, we have the following:

$$
\left|M_{\epsilon} y_{n}(t)\right| \leq c \quad \forall t \in[0, T] .
$$

From $(\mathrm{H} 2),(\mathrm{H} 3)$ and $(18)$, we see

$$
\left|C_{\epsilon} J_{\epsilon}^{M} y_{n}(t)\right| \leq c \quad \forall t \in[0, T] .
$$

Then in view of (14), (24) gives

$$
\left|M \frac{d}{d t} y_{n}(t)\right|^{2} \leq c+c\left|u_{n}\right|_{U}^{2}
$$

thus we see

$$
\left|M \frac{d}{d t} y_{n}(t)\right|_{T} \leq c,
$$

which implies

$$
\begin{gathered}
\left|M y_{n}(t)\right| \leq c \quad \forall t \in[0, T], \\
\left|\frac{d}{d t} y_{n}(t)\right|_{T} \leq c .
\end{gathered}
$$

Here, $|\cdot|_{T}$ is the norm in $L^{2}(0, T ; H)$. For every $m, n>0$

$$
\begin{aligned}
& \frac{d}{d t}\left|M^{1 / 2}\left(y_{m}-y_{n}\right)\right|^{2}+2\left(C_{\epsilon} J_{\epsilon}^{M}\left(y_{m}-y_{n}\right),\left(y_{m}-y_{n}\right)\right) \\
& \leq\left|u_{m}-u_{n}\right|_{U}^{2}+\left|y_{m}-y_{n}\right|^{2} .
\end{aligned}
$$

By some calculation, we see

$$
\left|M^{1 / 2}\left(y_{m}-y_{n}\right)\right|^{2} \leq c\left|u_{m}-u_{n}\right|_{T}^{2} \quad \forall t \in[0, T] .
$$

Hence $\left\{M^{1 / 2} y_{n}\right\}$ and $\left\{y_{n}\right\}$ are Cauchy sequences in $C([0, T] ; H)$. Note that $(\mathrm{H} 2)$; then there exists a function $\tilde{y} \in$ $C\left([0, T] ; D\left(M^{1 / 2}\right)\right)$ such that as $n \rightarrow \infty$

$$
y_{n} \longrightarrow \tilde{y} \text { strongly in } C([0, T] ; H) \cap L^{2}(0, T ; V),
$$

$M^{1 / 2} y_{n} \longrightarrow M^{1 / 2} \tilde{y} \quad$ strongly in $C([0, T] ; H) \cap L^{2}(0, T ; V)$.

This completes the proof.

Next, we define the approximation $g^{\epsilon}$ of $g$ and $h^{\epsilon}$ of $h$ as follows. For the details, we refer to [2-4]. Let

$$
g^{\epsilon}(t, y)=\int_{R^{N}} g\left(t, P_{N} y(s)-\epsilon \Lambda_{N} s\right) \rho(s) d s, \quad \epsilon>0 .
$$

Here, $\rho$ is a mollifier in $R^{N}, N=\left[\epsilon^{-1}\right] . P_{N}: L^{2} \rightarrow$ $X_{N}$ is the projection of $L^{2}(\Omega)$ on $X_{N}$, which is the finite dimensional space generated by $\left\{e_{i}\right\}_{i=1}^{N}$, where $\left\{e_{i}\right\}_{i=1}^{\infty}$ is an orthonormal basis in $L^{2}(\Omega) . \Lambda_{N}: R^{N} \rightarrow X_{N}$ is the operator defined by $\Lambda_{N}(s)=\sum_{i=1}^{N} s_{i} e_{i}, s=\left(s_{1}, \ldots, s_{N}\right)$.

We define $h_{\epsilon}: U \rightarrow \mathbb{R}:$

$$
\begin{array}{r}
h_{\epsilon}(y)=\inf \left\{\frac{\|y-x\|_{U}^{2}}{2 \epsilon}+h(x): x \in L^{2}(0, T ; U)\right\}, \\
\epsilon>0 .
\end{array}
$$

Now we define the penalty $L_{\epsilon}: L^{2}(0, T ; U) \rightarrow \mathbb{R}$ by

$$
\begin{aligned}
L_{\epsilon}(u)= & \int_{0}^{T}\left[g^{\epsilon}\left(t, y_{\epsilon}(t)\right)+h_{\epsilon}(u)\right] d t+\frac{1}{2}\left|u-u^{*}\right|_{L^{2}(0, T ; U)}^{2} \\
& +\frac{1}{2 \epsilon^{1 / 2}}\left[\epsilon^{1 / 2}+d_{S}\left(F\left(y_{\epsilon}(t)\right)\right)\right]^{2},
\end{aligned}
$$

where $y_{\epsilon}$ is the solution of $(14) \cdot d_{S}\left(F\left(y_{\epsilon}\right)\right)$ denotes the distance of $F\left(y_{\epsilon}\right)$ to $S$.

The approximating optimal control problems are as follows:

Minimize $L_{\epsilon}(u)$ over $u \in L^{2}(0, T ; U)$.

From Lemma 3, we easily show the following existence of the optimal solutions for $\left(\mathrm{P}^{\epsilon}\right)$ (see $\left.[2,3]\right)$. 
Theorem 4. $\left(P^{\epsilon}\right)$ has at least one optimal solution.

The following results are useful in discussing the approximating control problems.

Lemma 5. Let $u_{\epsilon} \rightarrow$ u weakly in $L^{2}(0, T ; U)$ as $\epsilon \rightarrow 0$. Then there exists a subsequence $\left\{y_{\epsilon}\right\}$, still denoted itself

$$
y_{\epsilon} \longrightarrow y \quad \text { strongly in } C([0, T] ; H) \cap L^{2}(0, T ; V) \text {, }
$$

as $\epsilon \rightarrow 0$, where $y_{\epsilon}$ is the solutions of (14) corresponding to $u_{\epsilon}$ and $y$ is the solutions of (10) corresponding to $u$.

Proof. Rewrite (14) as follows:

$$
\begin{aligned}
\frac{d M y_{\epsilon}(t)}{d t}+C_{\epsilon} J_{\epsilon}^{M} y_{\epsilon}(t) & =B u_{\epsilon}(t) \text { a.e in }(0, T), \\
y_{\epsilon}(0) & =y_{0} .
\end{aligned}
$$

Multiplying (35) by $J_{\epsilon}^{M} y_{\epsilon}(t)$, we see

$$
\begin{aligned}
& \frac{d}{d t}\left|M_{\epsilon}^{1 / 2} y_{\epsilon}(t)\right|^{2}+2\left(C_{\epsilon} J_{\epsilon}^{M} y_{\epsilon}(t), J_{\epsilon}^{M} y_{\epsilon}(t)\right) \\
& =2\left(B u_{\epsilon}, J_{\epsilon}^{M} y_{\epsilon}(t)\right) .
\end{aligned}
$$

Then, (H1)-(H3) yield

$$
\frac{d}{d t}\left|M_{\epsilon}^{1 / 2} y_{\epsilon}(t)\right|^{2} \leq c\left|M_{\epsilon}^{1 / 2} y_{\epsilon}(t)\right|^{2}+c\left|B u_{\epsilon}\right|^{2}
$$

Integrating the above inequality from 0 to $t(t \in(0, T])$ and using Gronwall's inequality, we have the following:

$$
\left|M_{\epsilon}^{1 / 2} y_{\epsilon}(t)\right|^{2} \leq c \quad \forall t \in[0, T],
$$

together with (18) implies

$$
\left|J_{\epsilon}^{M} y_{\epsilon}(t)\right|^{2} \leq c \quad \forall t \in[0, T]
$$

Since $|v|^{2}=\epsilon\left(M_{\epsilon} v, v\right)+\left(J_{\epsilon}^{M} v, v\right)$ for every $v \in H$, taking into account (36) (39), we see

$$
\left|y_{\epsilon}(t)\right|^{2} \leq c \quad \forall t \in[0, T]
$$

Multiplying (35) by $M_{\epsilon} J_{\epsilon}^{M} y_{\epsilon}(t)$, we see

$$
\begin{gathered}
\frac{d}{d t}\left|M_{\epsilon} y_{\epsilon}(t)\right|^{2}+2\left(C_{\epsilon} J_{\epsilon}^{M} y_{\epsilon}(t), M_{\epsilon} J_{\epsilon}^{M} y_{\epsilon}(t)\right) \\
=2\left(B u_{\epsilon}, M_{\epsilon} J_{\epsilon}^{M} y_{\epsilon}(t)\right) .
\end{gathered}
$$

Then we get the following:

$$
\frac{d}{d t}\left|M_{\epsilon} y_{\epsilon}(t)\right|^{2} \leq c\left|M_{\epsilon} y_{\epsilon}(t)\right|^{2}+c\left|u_{\epsilon}\right|_{U}^{2},
$$

from which it follows that

$$
\left|M_{\epsilon} y_{\epsilon}(t)\right| \leq c \quad \forall t \in[0, T]
$$

From (H2), (H3), and (18), we see

$$
\left|C_{\epsilon} J_{\epsilon}^{M} y_{\epsilon}(t)\right| \leq c .
$$

Then in view of (14) and (24) give

$$
\left|M \frac{d}{d t} y_{\epsilon}(t)\right|^{2} \leq c+c\left|u_{n}\right|_{U}^{2},
$$

Thus, we see

$$
\left|M \frac{d}{d t} y_{\epsilon}(t)\right|_{T} \leq c,
$$

which implies

$$
\begin{gathered}
\left|M y_{\epsilon}(t)\right| \leq c \quad \forall t \in[0, T], \\
\left|\frac{d}{d t} y_{\epsilon}(t)\right|_{T} \leq c .
\end{gathered}
$$

For every $m, n>0$,

$$
\begin{aligned}
& \frac{d}{d t}\left|M^{1 / 2}\left(y_{\epsilon_{m}}-y_{\epsilon_{n}}\right)\right|^{2} \\
& \quad+2\left(C_{\epsilon_{m}} J_{\epsilon_{m}}^{M} y_{\epsilon_{m}}-C_{\epsilon_{n}} J_{\epsilon_{n}}^{M} y_{\epsilon_{n}}, y_{\epsilon_{m}}-y_{\epsilon_{n}}\right) \\
& \leq\left|u_{\epsilon_{m}}-u_{\epsilon_{n}}\right|_{U}^{2}+\left|y_{\epsilon_{m}}-y_{\epsilon_{n}}\right|^{2},
\end{aligned}
$$

Using the identities $w=J_{\epsilon_{m}}^{M} w+\epsilon_{m} M_{\epsilon_{m}} w$ for every $w \epsilon$ $H$, and so forth, we see

$$
\begin{aligned}
&\left(C_{\epsilon_{m}} J_{\epsilon_{m}}^{M} y_{\epsilon_{m}}-C_{\epsilon_{n}} J_{\epsilon_{n}}^{M} y_{\epsilon_{n}}, y_{\epsilon_{m}}-y_{\epsilon_{n}}\right) \\
&=\left(C_{\epsilon_{m}} J_{\epsilon_{m}}^{M} y_{\epsilon_{m}}-C_{\epsilon_{n}} J_{\epsilon_{n}}^{M} y_{\epsilon_{n}}, J_{\epsilon_{m}}^{M} y_{\epsilon_{m}}-J_{\epsilon_{n}}^{M} y_{\epsilon_{n}}\right) \\
& \quad+\left(C_{\epsilon_{m}} J_{\epsilon_{m}}^{M} y_{\epsilon_{m}}-C_{\epsilon_{n}} J_{\epsilon_{n}}^{M} y_{\epsilon_{n}}, \epsilon_{m} M_{\epsilon_{m}} y_{\epsilon_{m}}-\epsilon_{n} M_{\epsilon_{n}} y_{\epsilon_{n}}\right) \\
& \geq \\
&\left(C_{\epsilon_{m}} J_{\epsilon_{m}}^{M} y_{\epsilon_{m}}-C_{\epsilon_{n}} J_{\epsilon_{n}}^{M} y_{\epsilon_{n}}, \epsilon_{m} C_{\epsilon_{m}} J_{\epsilon_{m}}^{M} y_{\epsilon_{m}}-\epsilon_{n} C_{\epsilon_{n}} J_{\epsilon_{n}}^{M} y_{\epsilon_{n}}\right) \\
& \quad+\left(C_{\epsilon_{m}} J_{\epsilon_{m}}^{M} y_{\epsilon_{m}}-C_{\epsilon_{n}} J_{\epsilon_{n}}^{M} y_{\epsilon_{n}}, \epsilon_{m} M_{\epsilon_{m}} y_{\epsilon_{m}}-\epsilon_{n} M_{\epsilon_{n}} y_{\epsilon_{n}}\right) \\
& \geq-c\left(\epsilon_{m}+\epsilon_{n}\right) .
\end{aligned}
$$

Because of (43) and (44), we obtain the following:

$$
\begin{aligned}
& \left|\frac{d}{d t} M^{1 / 2}\left(y_{\epsilon_{m}}-y_{\epsilon_{n}}\right)\right|^{2} \\
& \leq c\left|M^{1 / 2}\left(y_{\epsilon_{m}}-y_{\epsilon_{n}}\right)\right|^{2}+c\left|u_{\epsilon_{m}}-u_{\epsilon_{n}}\right|_{U}^{2} \\
& \quad+c\left(\epsilon_{m}+\epsilon_{n}\right),
\end{aligned}
$$

where $c$ is a constant independent of $m$ and $n$. Then Gronwall's inequality yields

$$
\left|M^{1 / 2}\left(y_{\epsilon_{m}}-y_{\epsilon_{n}}\right)\right|^{2} \leq c\left\{\left|u_{\epsilon_{m}}-u_{\epsilon_{n}}\right|_{T}^{2}+\left(\epsilon_{m}+\epsilon_{n}\right)\right\}
$$

$$
\forall t \in[0, T] .
$$


Hence, $\left\{M^{1 / 2} y_{\epsilon_{n}}\right\}$ and $\left\{y_{\epsilon_{n}}\right\}$ are Cauchy sequences in $C([0, T] ; H)$. Note that $(\mathrm{H} 2)$; then there exists a function $y \in C\left([0, T] ; D\left(M^{1 / 2}\right)\right) \cap L^{2}(0, T ; V)$ such that as $n \rightarrow \infty$, $\epsilon_{n} \rightarrow 0$

$y_{\epsilon_{n}} \longrightarrow y$ strongly in $C([0, T] ; H) \cap L^{2}(0, T ; V)$,

$M^{1 / 2} y_{\epsilon_{n}} \longrightarrow M^{1 / 2} y$ strongly in $C([0, T] ; H)$.

Thus, we deduce that as $\epsilon_{n} \rightarrow 0$,

$$
J_{\epsilon_{n}}^{M} y_{\epsilon_{n}} \longrightarrow y \text { strongly in } C([0, T] ; H),
$$

Note that

$M^{1 / 2} J_{\epsilon_{n}}^{M} y_{\epsilon_{n}} \longrightarrow M^{1 / 2} y \quad$ strongly in $C([0, T] ; H)$.

Indeed, we see

$$
\begin{aligned}
& \left|M^{1 / 2} J_{\epsilon_{n}}^{M} y_{\epsilon_{n}}-M^{1 / 2} y\right|^{2} \\
& \quad \leq 2\left|M^{1 / 2}\left(J_{\epsilon_{n}}^{M} y_{\epsilon_{n}}-y_{\epsilon_{n}}\right)\right|^{2}+\left|M^{1 / 2}\left(y_{\epsilon_{n}}-y\right)\right|^{2} \\
& \quad=-2 \epsilon_{n}\left(M_{\epsilon_{n}} y_{\epsilon_{n}}, M_{\epsilon_{n}} y_{\epsilon_{n}}-M y_{\epsilon_{n}}\right)+\left|M^{1 / 2}\left(y_{\epsilon_{n}}-y\right)\right|^{2} \\
& \quad \leq c \epsilon_{n}+\left|M^{1 / 2}\left(y_{\epsilon_{n}}-y\right)\right|^{2} \longrightarrow 0,
\end{aligned}
$$

for all $t \in[0, T]$. From (43) and (46), $\left\{M_{\epsilon_{n}} y_{\epsilon_{n}}\right\}$ is uniformly bounded and equicontinuous in $C([0, T] ; H)$. Hence the Ascoli-Arzela theorem gives that as $\epsilon_{n} \rightarrow 0$, for every $v \in$ $H,\left(M_{\epsilon_{n}} y_{\epsilon_{n}}, v\right) \rightarrow(M y, v)$ strongly in $C([0, T])$. In virtue of (46) and (48), weak closedness of $d / d t$, and $M$, it is shown that

$$
\begin{gathered}
\frac{d y_{\epsilon_{n}}}{d t} \longrightarrow \frac{d y}{d t} \quad \text { weakly in } L^{2}(0, T ; H), \\
\frac{M d y_{\epsilon_{n}}}{d t} \longrightarrow \frac{M d y}{d t} \quad \text { weakly in } L^{2}(0, T ; H) .
\end{gathered}
$$

Therefore, $y \in A C([0, T] ; D(M))$ and $d y / d t \in L^{2}(0$, $T ; D(M))$. By $A C([0, T] ; H])$, we denote the space of all $H$ valued strongly absolutely continuous functions on $[0, T]$. We easily get that $y(t) \in D(C)$ a.e. $t \in(0, T)$ and there exists a function $\xi \in L^{\infty}(0, T ; H)$ such that as $\epsilon_{n} \rightarrow 0$,

$$
C_{\epsilon_{n}} J_{\epsilon_{n}}^{M} y_{\epsilon_{n}} \longrightarrow \xi \quad \text { weakly star in } L^{\infty}(0, T ; H),
$$

and $\xi(t) \in C y=A y+\beta(y)$ a.e. $t \in(0, T)$. Thus, letting $\epsilon_{n} \rightarrow 0$ in (35), we see

$$
\begin{gathered}
\frac{d M y(t)}{d t}+A y+\xi(t)=B u(t) \quad \text { a.e in }(0, T), \\
y(0)=y_{0} .
\end{gathered}
$$

Lemma 6. Let $y_{0} \in D(M) \cap V, u \in L^{2}(0, T ; U)$; then $y_{\epsilon} \rightarrow y$ strongly in $C([0, T] ; H)$ as $\epsilon \rightarrow 0$, where $y_{\epsilon}$ is the solutions of (14) corresponding to $u$ and $y$ is the solutions of (1) corresponding to $u$ with the initial condition $y(0)=y_{0}$. Furthermore,

$$
\left|y_{\epsilon}-y\right|_{C([0, T] ; H)} \leq c \epsilon^{1 / 2} .
$$

Proof. By the same argument in the proof of Lemma 5, we have the following:

$$
y_{\epsilon} \longrightarrow y \text { strongly in } C([0, T] ; H) \cap L^{2}(0, T ; V) .
$$

We have for all $\epsilon$ and $\lambda$,

$$
\frac{d M\left(y_{\epsilon}(t)-y_{\lambda}(t)\right)}{d t}+C_{\epsilon} J_{\epsilon}^{M} y_{\epsilon}(t)-C_{\lambda} J_{\lambda}^{M} y_{\lambda}(t)=0
$$$$
\text { a.e in }(0, T) \text {, }
$$

$$
y_{\epsilon}(0)-y_{\lambda}(0)=0 .
$$

Multiplying (62) by $y_{\epsilon}(t)-y_{\lambda}(t)$, we have

$$
\begin{aligned}
& \frac{d\left|M^{1 / 2}\left(y_{\epsilon}(t)-y_{\lambda}(t)\right)\right|^{2}}{d t} \\
& \quad+2\left(C_{\epsilon} J_{\epsilon}^{M} y_{\epsilon}(t)-C_{\lambda} J_{\lambda}^{M} y_{\lambda}(t), y_{\epsilon}(t)-y_{\lambda}(t)\right)=0 .
\end{aligned}
$$

Using the identities $w=J_{\epsilon}^{M} w+\epsilon M_{\epsilon} w$ for every $w \in H$, and so forth, we get the following:

$$
\begin{aligned}
\left(C_{\epsilon} J_{\epsilon}^{M} y_{\epsilon}-C_{\lambda} J_{\lambda}^{M} y_{\lambda}, y_{\epsilon}-y_{\lambda}\right) \\
=\left(C_{\epsilon} J_{\epsilon}^{M} y_{\epsilon}-C_{\lambda} J_{\lambda}^{M} y_{\lambda}, J_{\epsilon}^{M} y_{\epsilon}-J_{\lambda}^{M} y_{\lambda}\right) \\
\quad+\left(C_{\epsilon} J_{\epsilon}^{M} y_{\epsilon}-C_{\lambda} J_{\lambda}^{M} y_{\lambda}, \epsilon M_{\epsilon} y_{\epsilon}-\lambda M_{\lambda} y_{\lambda}\right) \\
\geq-c(\epsilon+\lambda) .
\end{aligned}
$$

Thus, we see

$$
\frac{d\left|M^{1 / 2}\left(y_{\epsilon}(t)-y_{\lambda}(t)\right)\right|^{2}}{d t} \leq c(\epsilon+\lambda)
$$

then

$$
\left|M^{1 / 2}\left(y_{\epsilon}(t)-y_{\lambda}(t)\right)\right|_{C([0, T] ; H)}^{2} \leq c(\epsilon+\lambda) .
$$

Because of (61), letting $\lambda \rightarrow 0$ in (66), we get (60).

Lemma 7. Let $u_{\epsilon}$ be optimal for the problem $\left(P^{\epsilon}\right)$ and $y_{\epsilon}$ be the solution of (14) corresponding to $u_{\epsilon}$. For $\epsilon \rightarrow 0$, then

$$
y_{\epsilon} \longrightarrow y_{*} \quad \text { strongly in } C([0, T] ; H) \cap L^{2}(0, T ; V) \text {, }
$$

$$
u_{\epsilon} \longrightarrow u_{*} \text { strongly in } L^{2}(0, T ; U) \text {. }
$$

Proof. For any $\epsilon>0$, we have the following:

$$
\begin{aligned}
L_{\epsilon}\left(u_{\epsilon}\right) \leq & L_{\epsilon}\left(u_{*}\right) \\
= & \int_{0}^{T}\left[g^{\epsilon}\left(t, y_{\epsilon}(t)\right)+h_{\epsilon}\left(u_{*}(t)\right)\right] d t \\
& +\frac{1}{2 \epsilon^{1 / 2}}\left[\epsilon^{1 / 2}+d_{S}\left(F\left(y_{\epsilon}(t)\right)\right)\right]^{2} .
\end{aligned}
$$


By Lemma 5, we know $y_{\epsilon} \rightarrow y_{*}$ strongly in $C([0, T] ; H)$. So we have the following:

$$
\begin{gathered}
g^{\epsilon}\left(t, y_{\epsilon}\right) \longrightarrow g\left(t, y_{*}\right) \quad \forall t \in[0, T], \\
h_{\epsilon}\left(u_{\epsilon}\right) \longrightarrow h\left(u_{*}\right) .
\end{gathered}
$$

So

$$
\begin{aligned}
\lim _{\epsilon \rightarrow 0} \int_{0}^{T} g^{\epsilon}\left(t, y_{\epsilon}(t)\right) d t & =\int_{0}^{T} g\left(t, y_{*}(t)\right) d t, \\
\lim _{\epsilon \rightarrow 0} \int_{0}^{T} h_{\epsilon}\left(u_{*}(t)\right) d t & =\int_{0}^{T} h\left(u_{*}(t)\right) d t .
\end{aligned}
$$

Similarly, by (60) and (H5), we obtain the following:

$$
\begin{aligned}
& \frac{1}{2 \epsilon^{1 / 2}}\left[\epsilon^{1 / 2}+d_{S}\left(F\left(y_{\epsilon}\right)\right)\right]^{2} \\
& \quad \leq \frac{1}{2 \epsilon^{1 / 2}}\left[\epsilon^{1 / 2}+\left\|F\left(y_{\epsilon}\right)-F\left(y_{*}\right)\right\|_{Z}\right]^{2} \\
& \quad \leq c \epsilon \longrightarrow 0 \text { as } \epsilon \longrightarrow 0 .
\end{aligned}
$$

Then, we get the following:

$$
\limsup _{\epsilon \rightarrow 0} L_{\epsilon}\left(u_{\epsilon}\right) \leq L\left(u_{*}\right) \text {. }
$$

On the other hand, since $\left\{u_{\epsilon}\right\}$ is bounded in $L^{2}(0, T ; U)$, there exists $u_{1} \in L^{2}(0, T ; U)$ such that, on some subsequence $\epsilon$, still denoted by itself, as $\epsilon \rightarrow 0$,

$$
u_{\epsilon} \longrightarrow u_{1} \quad \text { weakly in } L^{2}(0, T ; U),
$$

and so, by Lemma 5 ,

$y_{\epsilon} \longrightarrow y_{1}=y\left(u_{1}\right) \quad$ strongly in $C(0, T ; H) \cap L^{2}(0, T ; V)$.

By (66), one can check easily that

$$
\frac{1}{2 \epsilon^{1 / 2}}\left[\epsilon^{1 / 2}+d_{S}\left(F\left(y_{\epsilon}\right)\right)\right]^{2} \leq c .
$$

Thus, $d_{S}\left(F\left(y_{\epsilon}\right)\right) \rightarrow 0$ as $\epsilon \rightarrow 0$. Since $S$ is closed and convex, $F\left(y_{1}\right)=\lim _{\epsilon \rightarrow 0} F\left(y_{\epsilon}\right) \in S$. Since the function $u \rightarrow$ $\int_{0}^{T} h(u) d t$ is weakly lower semicontinuous on $L^{2}(0, T ; U)$, we

$$
\liminf _{\epsilon \rightarrow 0} L_{\epsilon}\left(u_{\epsilon}\right) \geq L\left(u_{1}\right) \geq L\left(u_{*}\right) .
$$

Together with (72), we obtain

$$
\lim _{\epsilon \rightarrow 0} L_{\epsilon}\left(u_{\epsilon}\right)=L\left(u_{*}\right) .
$$

Therefore,

$$
\lim _{\epsilon \rightarrow 0} \int_{0}^{T}\left|u_{\epsilon}-u_{*}\right|_{U}^{2} d t=0
$$

Hence, $y_{1}=y_{*}, u_{1}=u_{*}$. This completes the proof.

\section{Necessary Condition on Optimality}

Let $\partial g$ the generalized gradient of $y \rightarrow g(t, y)$. Let $Y^{*}=$ $\left(H^{s}(\Omega)\right)^{\prime}+V^{\prime}$ which is the dual of $Y=H^{s}(\Omega) \cap V$ with $s>$ $N / 2$.

Firstly, we consider the following Cauchy problem:

$$
\begin{gathered}
\frac{d M p_{\epsilon}}{d t}-A p_{\epsilon}-\dot{\beta}^{\epsilon}\left(y_{\epsilon}\right) p_{\epsilon}-\left[F^{\prime}\left(y_{\epsilon}\right)\right]^{*} \xi_{\epsilon} \\
=\lambda_{\epsilon} \nabla g^{\epsilon}\left(t, y_{\epsilon}\right) \quad \text { in }(0 . T), \\
p_{\epsilon}(T)=0,
\end{gathered}
$$

where $\dot{\beta}^{\epsilon}=\left(\beta^{\epsilon}\right)^{\prime}, \beta_{\epsilon}=\epsilon^{-1}\left(I-(I+\epsilon \beta)^{-1}\right), \beta^{\epsilon}=\int_{-\infty}^{\infty}\left[\beta_{\epsilon}(r-\right.$ $\left.\left.\epsilon^{2} \theta\right)-\beta_{\epsilon}\left(-\epsilon^{2} \theta\right)\right] \rho(\theta) d \theta+\beta_{\epsilon}(0)$, and $\rho$ is a $C_{0}^{\infty}$-mollifier on $\mathbb{R}$.

Lemma 8. Problem (79) has a unique absolutely continuous function $p_{\epsilon} \in L^{2}(0, T ; V) \cap C([0, T] ; H)$ with $p_{\epsilon}^{\prime} \in L^{2}\left(0, T ; V^{\prime}\right)$, such that

$$
\left|p_{\epsilon}(t)\right|_{2}^{2}+\int_{0}^{T}\left\|p_{\epsilon}(t)\right\|_{V}^{2} d t \leq c \quad \forall \epsilon>0, t \in[0, T],
$$

$$
\int_{Q}\left|p_{\epsilon} \dot{\beta}^{\epsilon}\left(y_{\epsilon}\right)\right| d x d t \leq c \quad \forall \epsilon>0 .
$$

Proof. From (H1)-(H3) and $\dot{\beta}^{\epsilon}\left(y_{\epsilon}\right) \geq 0$, it is seen that $C=$ $M^{-1}\left(A+\dot{\beta}^{\epsilon}\left(y_{\epsilon}\right)\right): V \rightarrow V^{\prime}$ is demicontinuous monotone operator that satisfies

$$
\begin{gathered}
(C \omega, \omega) \geq w\|\omega\|^{p}+c \quad \forall \omega \in V, \\
\|C \omega\|_{*} \leq c\left(1+\|\omega\|^{p-1}\right),
\end{gathered}
$$

where $w>0$ and $p \geq 2$. It follows by Theorem $1.9^{\prime}$ of [4] that (79) has a unique solution $p_{\epsilon} \in L^{2}(0, T ; V) \cap C([0, T] ; H)$ with $p_{\epsilon}^{\prime} \in L^{2}\left(0, T ; V^{\prime}\right)$. Multiplying (79) by $J_{\epsilon}^{M} p_{\epsilon}(t)$ and using the self-adjointness of $M$ and integrating over $[t, T]$, we see

$$
\left|M_{\epsilon}^{1 / 2} p_{\epsilon}(t)\right|^{2}+w \int_{t}^{T}\left\|p_{\epsilon}(s)\right\|_{V}^{2} d s \leq c \int_{t}^{T}\left|M_{\epsilon}^{1 / 2} p_{\epsilon}(s)\right|^{2} d s+c,
$$

Because of $a\left|J_{\epsilon}^{M} p_{\epsilon}(t)\right|^{2} \leq\left|M^{1 / 2} J_{\epsilon}^{M} p_{\epsilon}(t)\right|^{2} \leq\left|M_{\epsilon}^{1 / 2} p_{\epsilon}(t)\right|^{2}$, $\left|\lambda_{\epsilon} \nabla g^{\epsilon}\left(t, \tilde{y}_{n}\right)\right|_{L^{\infty}(0, T ; H)} \leq c$ and $\left|\left[F^{\prime}\left(y_{\epsilon}\right)\right]^{*} \xi_{\epsilon}\right|_{L^{2}\left(0, T ; V^{\prime}\right)} \leq c$. And so by Gronwall's lemma we obtain the following:

$$
\left|M_{\epsilon}^{1 / 2} p_{\epsilon}(t)\right|_{2}^{2}+\int_{0}^{T}\left\|p_{\epsilon}(s)\right\|_{V}^{2} d s \leq c \quad \forall t \in[0, T] .
$$

Combining the above equalities, we see

$$
\left|J_{\epsilon}^{M} p_{\epsilon}(t)\right|_{2}^{2} \leq c \quad \forall t \in[0, T]
$$

Since $w=J_{\epsilon_{m}}^{M} w+\epsilon_{m}\left(M_{\epsilon_{m}} w, w\right)$ for every $w \in H$, taking into account the above equalities, we have the following:

$$
\left|p_{\epsilon}(t)\right|_{2}^{2} \leq c \quad \forall t \in[0, T] .
$$


Thus, we obtain (80).

Multiplying (79) by $\zeta\left(p_{\epsilon}\right)$ and integrate on $Q$, where $\zeta$ is a smooth monotonically increasing approximation of the sign function such that $\zeta(0)=0$. For instance

$$
\zeta=\zeta_{\lambda}(r)=\int_{-\infty}^{\infty}\left(\zeta_{\lambda}(r-\lambda \theta)-\zeta_{\lambda}(-\lambda \theta)\right) \rho(\theta) d \theta,
$$

where $\zeta_{\lambda}(r)=r|r|^{-1}$ for $|r| \geq \lambda, \zeta_{\lambda}(r)=\lambda^{-1} r$ for $|r|<\lambda$, and $\rho$ is a $C_{0}^{\infty}$-mollifier. Then $\left(A p_{\epsilon}(t), \zeta\left(p_{\epsilon}(t)\right)\right) \geq 0$; therefore,

$$
\int_{Q} \dot{\beta}^{\epsilon}\left(y_{\epsilon}\right) \zeta\left(p_{\epsilon}\right) p_{\epsilon} d x d t \leq \int_{Q}\left|\nabla_{y} g^{\epsilon}\left(t, y_{\epsilon}\right) \zeta\left(p_{\epsilon}\right)\right| d x d t
$$$$
\forall \epsilon>0 \text {. }
$$

Then, letting $\zeta$ tend to the sign function, we get (81).

We state the main results of the necessary conditions on optimality as follows.

Theorem 9. Suppose that $(H 1)-(H 7)$ hold. Let $\left(y_{*}, u_{*}\right)$ be an optimal pair of problem $(P)$. Then, there exists function $p \in L^{\infty}(0, T ; H) \cap L^{2}(0, T ; V) \cap B V\left([0, T] ; Y^{*}\right)$, a measure $\mu \in\left(L^{\infty}(Q)\right)^{\prime}, \lambda_{0} \in \mathbb{R}, \xi_{0} \in \mathbb{Z}^{*}$ satisfying

$$
\begin{gathered}
\frac{d}{d t} M p-A p-\mu-\left[F^{\prime}\left(y_{*}\right)\right]^{*} \xi_{0} \in L^{\infty}(0, T ; H), \\
\frac{d}{d t} M p(t)-A p(t)-\mu-\left[F^{\prime}\left(y_{*}\right)\right]^{*} \\
\times \xi_{0} \in \lambda_{0} \partial g\left(t, y_{*}\right) \quad \text { a.e.in }(0, T), \\
p(T)=0, \\
\left\langle\xi_{0}, w-F\left(y_{*}\right)\right\rangle \leq 0 \quad \forall w \in S, \\
B^{*} p \in \lambda_{0} \partial h\left(u_{*}(t)\right), \quad \text { a.e.t } \in(0, T), \\
\left(\lambda_{0}, \xi_{0}\right) \neq 0 .
\end{gathered}
$$

Proof. Since $\left(y_{\epsilon}, u_{\epsilon}\right)$ is optimal for problem $\left(\mathrm{P}^{\epsilon}\right)$, we see

$$
L_{\epsilon}\left(u_{\epsilon}^{\rho}\right) \geq L_{\epsilon}\left(u_{\epsilon}\right) \quad \text { for any } \rho>0, v \in L^{2}(0, T ; V) .
$$

Here $u_{\epsilon}^{\rho}=u_{\epsilon}+\rho v$. Thus,

$$
\frac{L_{\epsilon}\left(u_{\epsilon}^{\rho}\right)-L_{\epsilon}\left(u_{\epsilon}\right)}{\rho} \geq 0 .
$$

By some calculation, we have the following:

$$
\begin{gathered}
\lim _{\rho \rightarrow 0} \int_{0}^{T} \frac{g^{\epsilon}\left(t, y_{\epsilon}^{\rho}(t)\right)-g^{\epsilon}\left(t, y_{\epsilon}(t)\right)}{\rho} \\
\quad=\int_{0}^{T}\left(\nabla g^{\epsilon}\left(t, y_{\epsilon}(t)\right), z_{\epsilon}\right) d t,
\end{gathered}
$$

where $z_{\epsilon} \in C([0, T] ; H) \cap L^{2}(0, T ; V) \cap W^{1,2}([0, T] ; H)$ is the following solution to the linear equation

$$
\begin{gathered}
\frac{d M z}{d t}+A z+\dot{\beta}^{\epsilon}\left(y_{\epsilon}\right) z=B v \text { in }(0, T), \\
z(0)=0 .
\end{gathered}
$$

Hence, we also have the following:

$$
\begin{gathered}
\lambda_{\epsilon}\left[\int_{0}^{T}\left\langle\nabla g^{\epsilon}\left(t, y_{\epsilon}\right), z_{\epsilon}\right\rangle d t+\int_{0}^{T}\left\langle\nabla h_{\epsilon}\left(u_{\epsilon}\right), v\right\rangle d t\right] \\
+\left\langle\xi_{\epsilon}, F^{\prime}\left(y_{\epsilon}\right) z_{\epsilon}\right\rangle \geq \int_{0}^{T}\left\langle u_{*}-u_{\epsilon}, v\right\rangle d t
\end{gathered}
$$

where

$$
\begin{gathered}
\lambda_{\epsilon}=\frac{\epsilon^{1 / 2}}{d_{S}\left(F\left(y_{\epsilon}\right)\right)+\epsilon^{1 / 2}}, \\
\xi_{\epsilon}= \begin{cases}\nabla d_{S}\left(F\left(y^{\epsilon}\right)\right), & \text { if } F\left(y^{\epsilon}\right) \notin S, \\
0 & \text { otherwise, }\end{cases}
\end{gathered}
$$

and $\xi_{\epsilon} \in \partial d_{S}\left(F\left(y^{\epsilon}\right)\right)$. Since $S$ is convex and closed, we see

$$
\begin{gathered}
\left\|\xi_{\epsilon}\right\|_{Z^{*}}= \begin{cases}1, & \text { if } F\left(y^{\epsilon}\right) \notin S, \\
0 & \text { otherwise, }\end{cases} \\
1 \leq \varphi_{\epsilon}^{2}+\left\|\xi_{\epsilon}\right\|_{Z^{*}}^{2} \leq 2
\end{gathered}
$$

So, we see

$$
\lambda_{\epsilon} \longrightarrow \lambda_{0}, \quad \xi_{\epsilon} \longrightarrow \xi_{0} \text { weakly in } Z^{*} .
$$

It follows from Lemma 7 that $y_{\epsilon} \rightarrow y_{*}$ strongly in $C([0, T] ; H) \cap L^{2}(0, T ; V)$. By the same arguments as those in [2-4], there exists $p \in C([0, T] ; H) \cap L^{2}(0, T ; V) \cap$ $B V\left([0, T] ; Y^{*}\right)$ and $\mu \in\left(L^{\infty}(Q)\right)^{*}$ such that, on some subsequence $\epsilon$, still denoted itself

$$
p_{\epsilon}(t) \longrightarrow p \text { strongly in } Y^{*}, \quad \forall t \in[0, T],
$$

where $B V\left([0, T] ; Y^{*}\right)$ is the space of all $Y^{*}$-valued functions $p:[0, T] \rightarrow Y^{*}$ with bounded variation on $[0, T]$. On the other hand, by (80), we see

$$
\begin{array}{r}
p_{\epsilon} \longrightarrow p \quad \text { weakly star in } L^{\infty}(0, T ; H), \\
\text { weakly in } L^{2}(0, T ; V) .
\end{array}
$$

Note that $V \hookrightarrow H$ is compact, for every $\lambda>0$, there is $\delta(\lambda)>0$ such that

$$
\begin{gathered}
\left|p_{\epsilon}(t)-p(t)\right|_{2} \leq\left\|p_{\epsilon}(t)-p(t)\right\|_{V}+\delta(\lambda)\left\|p_{\epsilon}(t)-p(t)\right\|_{Y^{*}} \\
\forall t \in[0, T] .
\end{gathered}
$$

This yields

$$
\begin{gathered}
p_{\epsilon} \longrightarrow p \quad \text { strongly in } L^{2}(0, T ; H), \\
p_{\epsilon}(t) \longrightarrow p(t) \quad \text { weakly in } H \forall t \in[0, T] .
\end{gathered}
$$

Moreover, by (81) we infer that there is $\mu \in\left(L^{\infty}(Q)\right)^{*}$ such that, on some generalized subsequence $\epsilon$,

$$
\begin{gathered}
\dot{\beta}^{\epsilon}\left(y_{\epsilon}\right) p_{\epsilon} \longrightarrow \mu \quad \text { weakly star in }\left(L^{\infty}(Q)\right)^{*}, \\
\left.\nabla g^{\epsilon}\left(t, y_{\epsilon}\right) \longrightarrow \eta \quad \text { weakly star in } L^{\infty}(0, T ; H)\right)^{*}, \\
\eta(t) \in \partial g\left(t, y_{*}\right) \quad \text { a.e. } t \in(0, T) .
\end{gathered}
$$


$Z$,

Since $F$ is continuously differentiable from $L^{2}(0, T ; V)$ to

$$
\left[F^{\prime}\left(y_{\epsilon}\right)\right]^{*} \xi_{\epsilon} \longrightarrow\left[F^{\prime}\left(y_{*}\right)\right]^{*} \xi_{0} \quad \text { weakly } L^{2}\left(0, T ; V^{\prime}\right) .
$$

Now letting $\epsilon \rightarrow 0$ in (79), it follows that

$$
\begin{gathered}
\frac{d}{d t} M p-A p-\mu-\left[F^{\prime}\left(y_{*}\right)\right]^{*} \xi_{0} \in L^{\infty}(0, T ; H), \\
\frac{d}{d t} M p(t)-A p(t)-\mu-\left[F^{\prime}\left(y_{*}\right)\right]^{*} \\
\times \xi_{0} \in \lambda_{0} \partial g\left(t, y_{*}\right) \quad \text { a.e. in }(0, T), \\
p(T)=0 .
\end{gathered}
$$

It follows from (93), (94), and (79) that

$$
\begin{aligned}
-\int_{0}^{T}\left\langle B^{*} p_{\epsilon}, v\right\rangle d t+\lambda_{\epsilon} \int_{0}^{T}\left\langle\nabla h_{\epsilon}\left(u_{\epsilon}\right), v\right\rangle d t \\
\quad \geq \int_{0}^{T}\left\langle u_{*}-u_{\epsilon}, v\right\rangle d t, \quad \forall v \in L^{2}(0, T ; V) .
\end{aligned}
$$

By Lemma 7, $u_{\epsilon} \rightarrow u_{*}$ strongly in $L^{2}(0, T ; U)$, it follows

$$
\int_{0}^{T}\left\langle\nabla h_{\epsilon}\left(u_{\epsilon}\right), v\right\rangle d t \longrightarrow \int_{0}^{T}\langle\nabla \zeta(t), v\rangle d t
$$

$\zeta(t) \in \partial h\left(u_{*}\right) \quad$ a.e. in $(0, T), \forall v \in L^{2}(0, T ; V)$.

Thus,

$$
\begin{array}{r}
-\int_{0}^{T}\left\langle B^{*} p, v\right\rangle d t+\lambda_{0} \int_{0}^{T}\langle\zeta(t), v\rangle d t \geq 0, \\
\forall v \in L^{2}(0, T ; V) .
\end{array}
$$

Since $\xi_{\epsilon} \in d_{S}\left(F\left(y_{\epsilon}\right)\right)$, we get $\left\langle\xi_{\epsilon}, w-F\left(y_{\epsilon}\right)\right\rangle \leq 0$ for all $w \in S$. Now we claim that $\left(\lambda_{0}, \xi_{0}\right) \neq 0$. Indeed, if $\lambda_{0}=0$, we have that $\left\{\xi_{\epsilon}\right\}$ is bounded in $Z^{*}$. By (H3), $S$ has finite codimentionality, so dose $S-F\left(y^{*}\right)$. Thus, it follows that $\xi_{\epsilon} \rightarrow \xi_{0}$ weakly in $Z^{*}$ and

$$
\left\langle\xi_{0}, w-F\left(y^{*}\right)\right\rangle \leq 0 \quad \forall w \in S .
$$

Finally, if $\left(\lambda_{0}, p\right)=0$, it follows from (105) that $\mu+$ $\left[F^{\prime}\left(y^{*}\right)\right]^{*} \xi_{0}=0$. So in the case that $\mu \notin R\left(\left[F^{\prime}\left(y^{*}\right)\right]^{*}\right)$, we must have $\left(\lambda_{0}, p\right) \neq 0$. Together with (104), (105), and (109), we completes the proof.

\section{Some Examples}

In this section, we present two examples.

Example 1. Consider the initial value controlled system

$$
\begin{gathered}
y_{t}-y_{x x t}+y y_{x}+\beta(y) \ni B u \quad \text { in }(0,1) \times[0, T], \\
y(0, t)=y(1, t)=0 \quad t \in[0, T], \\
y(x, 0)=y_{0} \quad \text { in }(0,1),
\end{gathered}
$$

where $1 y=y(x, t)$ is a function on $\mathbb{R} \times[0, T]$ and $\beta(\cdot)$ is a multivalued function on $\mathbb{R}$.

If $\beta(y)=0$, rewrite (110) in the form

$$
\begin{gathered}
y_{t}-y_{x x t}+y y_{x}=B u \quad \text { in }(0,1) \times[0, T], \\
y(0, t)=y(1, t)=0 \quad t \in[0, T], \\
y(x, 0)=y_{0} \quad \text { in }(0,1) .
\end{gathered}
$$

(111) was introduced by Benjamin et al. [23] as an approximate equation of the propagation of one-dimensional waves of small amplitude in water. If $y_{x} \geq 0, \beta(\cdot)$ satisfies (H3). $y_{0} \in$ $H^{2}((0,1)) \cap H_{0}^{1}((0,1))$. Since $\beta_{\epsilon}$ is a Lipschitz continuous and monotone increasing function, integration by parts yields

$$
\int_{\mathbb{R}} \beta_{\epsilon}(y)\left(I-\frac{d^{2}}{d x^{2}}\right) y d x \geq 0 \text { for every } y \in H^{2}(\mathbb{R}) .
$$

Thus, $C(=A+\beta)$ is m-accretive in $H$. We easily proof the following result.

Theorem 10. Suppose that $(H 1)-(H 7)$ hold. Let $\left(y_{*}, u_{*}\right)$ be an optimal pair of problem $(P)$. Then there exists function $p \in L^{\infty}(0, T ; H) \cap L^{2}(0, T ; V) \cap B V\left([0, T] ; Y^{*}\right)$, a measure $\mu \in\left(L^{\infty}(Q)\right)^{\prime}$ and $R$ with $\lambda_{0}, \xi_{0}$ satisfying

$$
\begin{gathered}
\left(I-\frac{d^{2}}{d x^{2}}\right) \frac{d}{d t} p-y p_{x}-\mu-\left[F^{\prime}\left(y_{*}\right)\right]^{*} \\
\times \xi_{0} \in L^{\infty}(0, T ; H), \\
\left(I-\frac{d^{2}}{d x^{2}}\right) \frac{d}{d t} p-y p_{x}-\mu-\left[F^{\prime}\left(y_{*}\right)\right]^{*} \\
\times \xi_{0} \in \lambda_{0} \partial g\left(t, y_{*}\right) \quad \text { a.e.in }(0,1) \times[0, T], \\
p(0, t)=p(1, t)=0 \quad \text { in }[0, T] \\
p(x, T)=0 \quad \text { in }(0,1) . \\
\left\langle\xi_{0}, w-F\left(y_{*}\right)\right\rangle \leq 0 \quad \forall w \in S, \\
B^{*} p \in \lambda_{0} \partial h\left(u_{*}\right)(t), \quad \text { a.e.t } \in(0, T), \\
\left(\lambda_{0}, \xi_{0}\right) \neq 0 .
\end{gathered}
$$

Example 2. Consider the initial boundary value controlled system

$$
\begin{gathered}
(I-\Delta) \frac{d y(x, t)}{d t}-\Delta y(x, t)+\beta(y(x, t)) \ni B u(x, t) \\
\text { in } \Omega \times[0, T], \\
y(x, t)=0 \quad \text { on } \partial \Omega \times[0, T], \\
y(x, 0)=y_{0} \quad \text { in } \Omega,
\end{gathered}
$$

where $\Omega \subset \mathbb{R}^{N}$ is a bounded domain with smooth boundary. $y_{0} \in H_{0}^{1}(\Omega) \cap H^{2}(\Omega), \beta(\cdot)$ satisfies $(\mathrm{H} 3) . M y=(I-\Delta) y$ with 
$D(M)=H_{0}^{1}(\Omega) \cap H^{2}(\Omega), A y=-\Delta y$. Since $\beta_{\epsilon}$ is a monotone function,

$$
\int_{\Omega} \beta_{\epsilon}(y)(-\Delta y) d x \geq 0, \quad \text { for every } y \in H_{0}^{2}(\Omega) \cap H^{2}(\Omega) .
$$

Then, $C(=A+\beta)$ is $\mathrm{m}$-accretive in $H$. We easily obtain similar necessary condition of optimality of problem (P).

\section{Conflict of Interests}

The authors declare that there is no conflict of interests regarding the publication of this paper.

\section{Acknowledgments}

The work is supported by the NNSFC (Grants nos. 10671211, 11126170), Hunan Provincial Natural Science Foundation of China (Grant no. 11JJ4006), and Doctor Priming Fund Project of University of South China (Grant no. 5-2011-XQD008).

\section{References}

[1] X. J. Li and J. M. Yong, Optimal Control Theory for InfiniteDimensional Systems, Birkhäauser, Boston, Mass, USA, 1995.

[2] G. S. Wang, "Optimal control of parabolic differential equations with two point boundary state constraints," SIAM Journal on Control and Optimization, vol. 38, no. 5, pp. 1639-1654, 2000.

[3] G. S. Wang, "Optimal control of parabolic variational inequality involving state constraint," Nonlinear Analysis: Theory, Methods \& Applications, vol. 42, no. 5, pp. 789-801, 2000.

[4] V. Barbu, Analysis and Control of Nonlinear Infinite-Dimensional Systems, Academic Press, Boston, Mass, USA, 1993.

[5] M. Tsutsumi and T. Matahashi, "On some nonlinear pseudoparabolic equations," Journal of Differential Equations, vol. 32, no. 1, pp. 65-75, 1979.

[6] W. Liu, Elementary Feedback Stabilization of the Linear Reaction-convection-diffusion Equation and the Wave Equation, vol. 66 of Mathmatiques et Applications, Springer, Berlin, Germany, 2010.

[7] S. I. Lyashko, Generalized Control in Linear Systems, Naukova Dumka, Kiev, Ukraine, 1998 (Russian).

[8] G. A. Sviridyuk and V. E. Fedorov, Linear Sobolev Type Equations and Degenerate Semigroups of Operators, VSP International Science, 2003.

[9] V. E. Fedorov and M. V. Plekhanova, "Optimal control of Sobolev type linear equations," Differential Equations, vol. 40, no. 11, pp. 1627-1637, 2004.

[10] L. W. White, "Control problems governed by a pseudoparabolic partial differential equation," Transactions of the American Mathematical Society, vol. 250, pp. 235-246, 1979.

[11] L. W. White, "Convergence properties of optimal controls of pseudoparabolic problems," Applied Mathematics and Optimization, vol. 7, no. 2, pp. 141-147, 1981.

[12] I. Bock and J. Lovíšek, "On pseudoparabolic optimal control problems," Kybernetika, vol. 29, no. 3, pp. 222-230, 1993.

[13] K. Kunisch and D. Wachsmuth, "Path-following for optimal control of stationary variational inequalities," Computational Optimization and Applications, vol. 51, no. 3, pp. 1345-1373, 2012.
[14] J. C. de Los Reyes, "Optimal control of a class of variational inequalities of the second kind," SIAM Journal on Control and Optimization, vol. 49, no. 4, pp. 1629-1658, 2011.

[15] K. Ito and K. Kunisch, "Optimal control of parabolic variational inequalities," Journal de Mathématiques Pures et Appliquées, vol. 93, no. 4, pp. 329-360, 2010.

[16] K. Ito and K. Kunisch, "An augmented Lagrangian technique for variational inequalities," Applied Mathematics and Optimization, vol. 21, no. 3, pp. 223-241, 1990.

[17] K. Ito and K. Kunisch, "Optimal control of elliptic variational inequalities," Applied Mathematics and Optimization, vol. 41, no. 3, pp. 343-364, 2000.

[18] X. M. Guo and S. X. Zhou, "Optimal control of parabolic variational inequalities with state constraint," Applied Mathematics and Mechanics (English Edition), vol. 24, no. 7, pp. 756-762, 2003.

[19] D. R. Adams and S. Lenhart, "Optimal control of the obstacle for a parabolic variational inequality," Journal of Mathematical Analysis and Applications, vol. 268, no. 2, pp. 602-614, 2002.

[20] E. Casas, "Pontryagin's principle for state-constrained boundary control problems of semilinear parabolic equations," SIAM Journal on Control and Optimization, vol. 35, no. 4, pp. 12971327, 1997.

[21] Z.-X. He, "State constrained control problems governed by variational inequalities," SIAM Journal on Control and Optimization, vol. 25, no. 5, pp. 1119-1144, 1987.

[22] H. Brézis, "Quelques Propriétés des Opérateurs Monotones et des Semi-groupes Non Linéaires," in Nonlinear operators and the calculus of variations, vol. 543 of Lecture Notes in Mathematics, pp. 56-82, Springer, Berlin, Germany, 1976.

[23] T. B. Benjamin, J. L. Bona, and J. J. Mahony, "Model equations for long waves in nonlinear dispersive systems," Philosophical Transactions of the Royal Society of London A, vol. 272, no. 1220, pp. 47-78, 1972. 


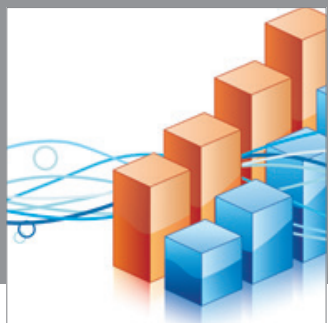

Advances in

Operations Research

mansans

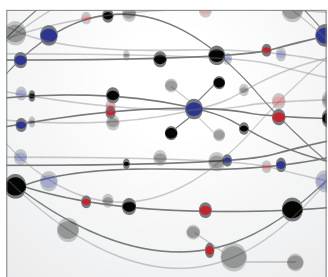

The Scientific World Journal
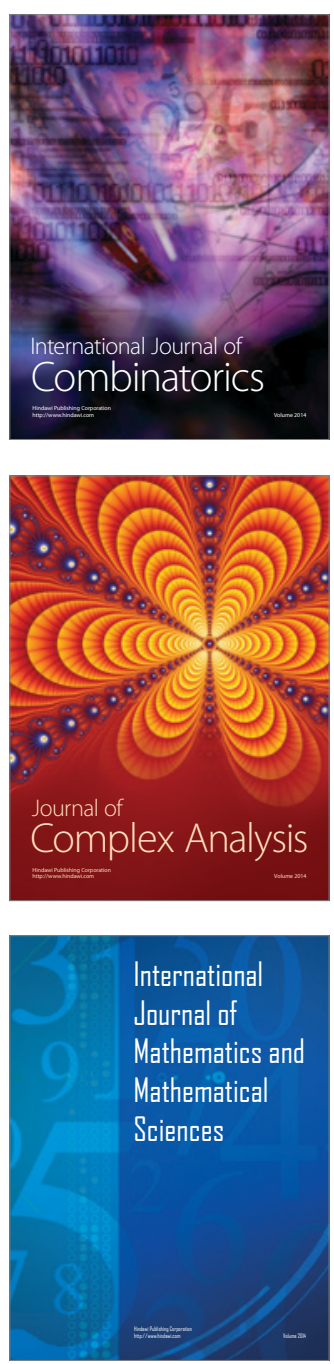
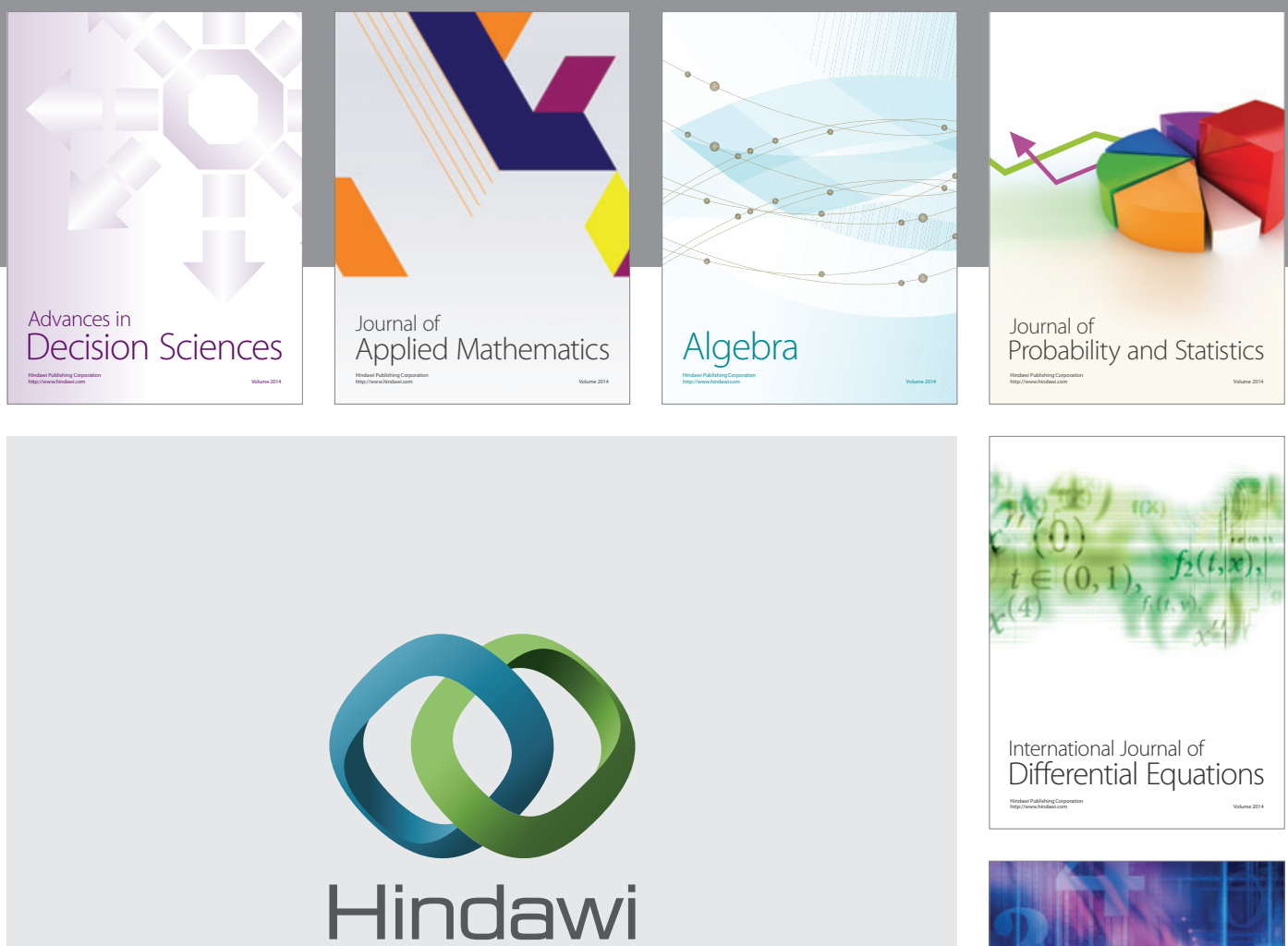

Submit your manuscripts at http://www.hindawi.com
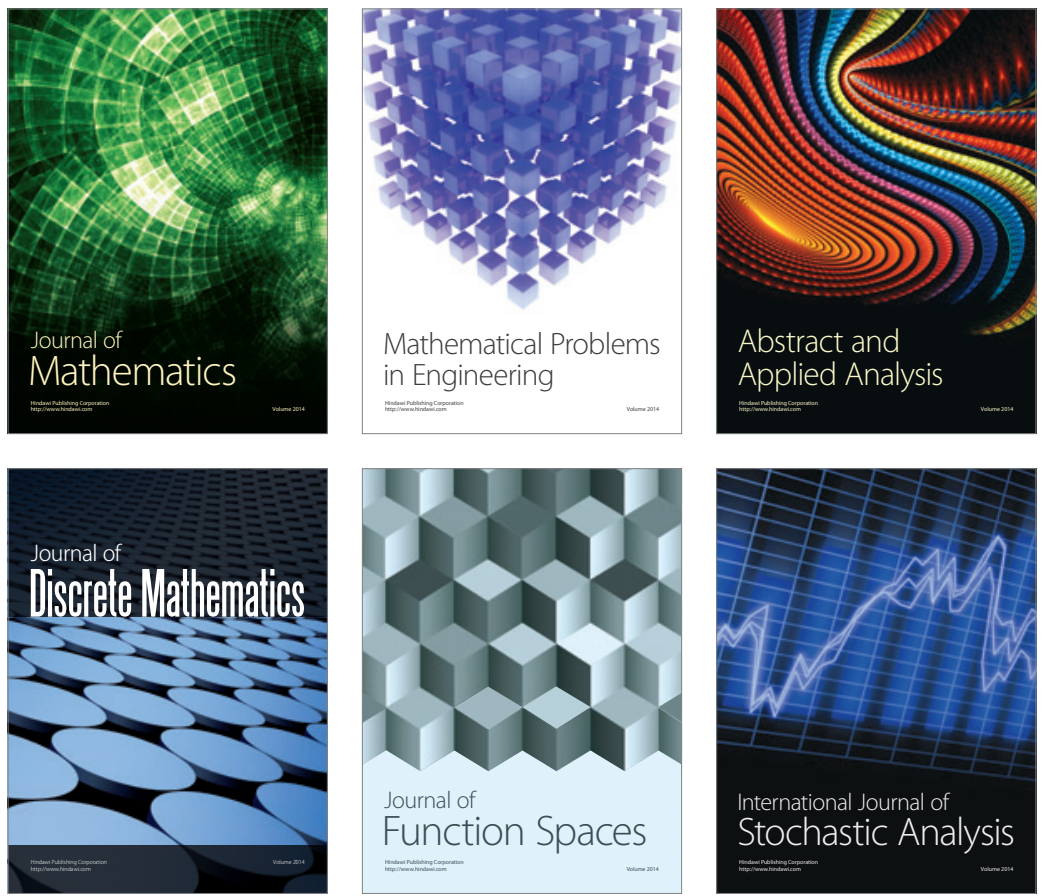

Journal of

Function Spaces

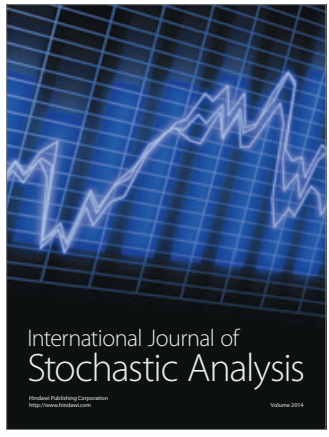

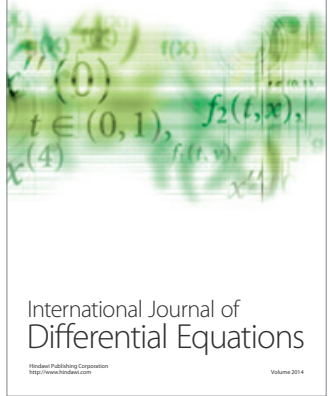
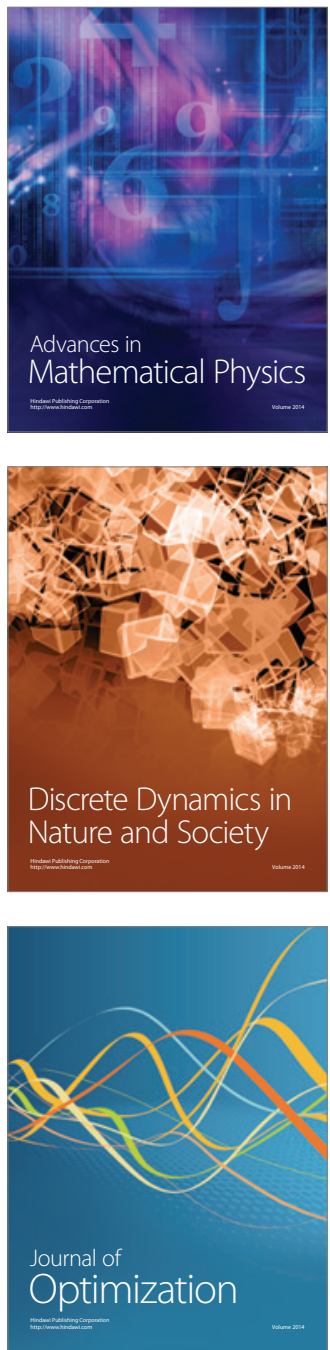